\title{
Asthma Phenotypes
}

\section{Steve Handoyo, MD, MPH, and Lanny J. Rosenwasser, MD}

\author{
Corresponding author \\ Lanny J. Rosenwasser, MD \\ Children's Mercy Hospitals and Clinics, 2401 Gillham Road, \\ Kansas City, MO 64111, USA. \\ E-mail: Irosenwasser@cmh.edu \\ Current Allergy and Asthma Reports 2009, 9:439-445 \\ Current Medicine Group LLC ISSN 1529-7322 \\ Copyright $@ 2009$ by Current Medicine Group LLC
}

The many roads leading to the syndrome of asthma have proven to be intricately interconnected. The chronic inflammation of asthma is characterized by airway hyperreactivity and variable reversibility. Past classification systems relied on assessment of daily impairment and the distinction between intrinsic (nonallergic) and extrinsic (allergic). With more precise asthma phenotypes, association studies likely will have greater significance. In addition, patients at higher risk for severe disease can be more effectively managed, and treatments can be directed to responders. In this review, we look at eight identified phenotypes: atopic and nonatopic status, pre-asthma wheezing, inflammatory mediator predominance, aspirin-sensitive, exercise-induced, severe, and flareprone asthma. Although significant overlap exists among the distinctions, any future phenotype classification system will need to incorporate these eight clinical asthmatic populations.

\section{Introduction}

The core definition of asthma has been unchanged for some time. The most recent summary of the National Asthma Education and Prevention Program Expert Panel succinctly describes asthma as "a complex disorder characterized by variable and recurring symptoms, airflow obstruction, bronchial hyperresponsiveness, and an underlying inflammation" [1]. Yet asthma has proven to be a complex interplay of genetic predisposition and temporally specific environmental exposures. One recent study suggests a link between the 17q21 locus and asthma that may be most pronounced for children younger than 4 years of age with smoke exposure [2]. Our challenge is to find clear distinctions within this diverse population grouped under the umbrella of asthma.

As our ability to process genetic data threatens to outpace our capacity to analyze them, it becomes increasingly clear that without successfully distinguishing the clinical phenotypes of asthma, we will continue to struggle with therapeutic outcomes that reflect unmet needs. The surprising failure of directed anti-interleukin (IL)-5 therapy to affect clinical outcomes despite successfully lowering eosinophilic counts initially cast doubt on the role of eosinophils in asthma [3]. New classifications of inflammatory markers in forced sputum now suggest subtypes of eosinophilic, neutrophilic, and paucigranulocytic asthma [4]. Two subsequent randomized, double-blind control trials looking at eosinophilic-predominant severe asthma patients showed a significant reduction in flares $[5,6]$. These studies highlight the importance of assigning phenotype for appropriate management.

In addition to observing asthma cohorts such as the Tucson and ALSPAC (Avon Longitudinal Study of Parents and Children) studies, authors have used various statistical analyses to identify asthma phenotypes. Using cluster analysis, one study examined mildly affected patients managed by primary care and more refractory asthma patients seen in specialty clinics [7]. For the patients with milder asthma, three phenotypes were appreciated: earlyonset atopic, obese noneosinophilic, and benign. The refractory group had those initial clusters plus significant early onset; symptom predominant; noneosinophilic; and late-onset, eosinophilic, minimal-symptom clusters. Comparing these clusters to a cohort that was managed by sputum-guided therapy, the authors suggest that by classifying phenotype, symptom- or sputum-based guidance can improve asthma control.

Previous attempts at asthma classification have been hampered by the extensive overlap between the various phenotypes. In this review, we discuss eight main phenotypic determinants that must be considered in any classification system. We address atopic and nonatopic asthma and the clinical attributes of inflammatory mediator-specific asthmatics. We also discuss pre-asthma wheezing as well as exacerbation prone and irreversible airway limitation. Potential triggers for asthma, including aspirin (ASA) sensitivity, infection, and exercise, likewise are examined. Finally, we address severe asthma and the flare-prone phenotype.

\section{Atopy}

Although the most traditional descriptions of asthma delineate allergic from nonallergic asthma, even this dichotomy appears controversial. Allergic asthma can be defined by positivity to skin prick test or specific IgE, though the pres- 
ence of sensitivity alone cannot prove causation of asthma without challenge. Additionally, nonatopic asthma patients may encompass diverse groups such as infection-triggered and ASA-sensitive asthma. Multiple studies have attempted to estimate the attributable risk of atopy for asthma, with a surprisingly low population-attributable risk of $33 \%$ to $56.3 \%$ [8]. Attempts to distinguish nonatopic and atopic asthma by immune response and pathology also have been equivocal $[9,10]$.

Atopic asthma patients likely account for slightly more than $50 \%$ of asthmatics. These patients tend to be younger yet still make up a large proportion of adult asthma patients [11]. Atopic asthma patients are more likely male with better response to steroids and greater forced expiratory volume in 1 second $\left(\mathrm{FEV}_{1}\right)$ [12]. Paroxysmal sneezing and seasonal rhinorrhea are more common in atopic asthma, although chronic rhinitis symptoms are seen in both [11]. Data analyzed from the Third National Health and Nutrition Examination Survey suggest that four allergens are associated with most atopic asthma patients: Alternaria spp, cat, and white oak, with inverse association with perennial rye [8].

Nonatopic asthma patients likely make up a more disparate group. These individuals do not have evidence of skin prick positivity or elevated IgE. More likely female and older, nonatopic asthma patients tend to have more severe disease and recent steroid requirement [11]. Some of this severity may be a result of the contribution of ASA-sensitive asthma patients, and nasal polyposis is more common in this group. Nonatopic asthma patients are more commonly triggered by infection, irritants, gastroesophageal reflux disease, stress, and exercise [12]. Various studies indicate a neutrophilic and an eosinophilic dominance in nonatopic asthma patients $[10,12]$.

Studies have examined the T-regulatory-cell response as well as pathologic findings in atopic and nonatopic asthma. Sputum neutrophils were found in significantly higher numbers in atopic compared with nonatopic asthma patients [10]. Chemokines and $\mathrm{CD}^{+} \mathrm{CD} 25^{+}$ T-regulatory cells were not statistically different in nonatopic and atopic asthma patients [9]. Airway pathology was similar for nonatopics with multitrigger wheezing and atopics with equal epithelial loss and thickened basement membranes [13]. These immunologic and pathologic findings, coupled with the unexpectedly low attributable risk of atopy in asthma in a few studies indicate that the delineation between atopic and nonatopic asthma may be overstated [14].

\section{Pre-asthma Wheezing}

Multiple prospective trials have observed newborns through childhood, adolescence, and even to adulthood in some cases in hopes of identifying risk factors for future asthma [15-17]. Several potential prediagnosis phenotypes have emerged. Complicating the diagnosis of asthma is the frequency of young children who have wheezing early in life, as late as age 3 years, but who are asymptomatic by age 6 years. These children may have virus-induced wheezing not associated with later asthma, and some may have smaller airways predisposed to wheezing that remits with growth [17]. Several recent studies identified potentially unique phenotypes in younger children [15-17].

Never or infrequent wheeze made up $50 \%$ to $59.3 \%$ of birth cohorts [16-18]. This group includes almost half of the children, which is in contrast to the US lifetime prevalence of asthma of $11.2 \%$ as reported by the Centers for Disease Control and Prevention. The infrequent/ never-wheeze population had similar IgE levels and atopy compared with those with transient early wheeze [17]. The infrequent wheeze phenotype did have greater $\mathrm{FEV}_{1}$ values, greater forced expiratory flow $(\mathrm{FEF})_{25 \%-75 \%}$, and lower responsiveness than the never-wheeze group [16].

Transient early wheeze, defined as those who experienced wheezing in the first 1.5 to 3 years but who were asymptomatic by age 4 to 6 years, comprises $16 \%$ to $20 \%$ of these cohorts. Although the Tucson study identified one group for early transient wheeze asymptomatic by age 6 years, the ALSPAC study delineated two groups, early- $(16.3 \%)$ and late-transient $(9 \%)$ wheeze with symptoms at age 18 months that was declining by ages 42 and 69 months, respectively [16,17]. Neither group showed significant association with atopy but did have some association with airway hyperreactivity, decreased $\mathrm{FEV}_{1}$, and $\mathrm{FEF}_{25 \%-75 \%}$ at 7 to 8 years, and maternal asthma and smoking. These studies identified a population that may have symptoms from viral infection or congenital small airways but is unlikely to have asthma symptoms that persist into later childhood.

Henderson et al. [16] calculated an intermediate wheeze phenotype-making up only $2.7 \%$-who have minimal symptoms at 18 months but significant prevalence by 42 months. Interestingly, this population had the strongest association with atopy in the ALSPAC study (OR, 8.26; 95\% CI, 5.24-13.36), specifically to Dermatophagoides pteronyssinus and cat allergen [16]. Intermediate wheeze also has the most significant association with airway hyperresponsiveness. This group is incorporated into persistent wheeze by the Tucson study and represents a likely pre-asthma state [17].

Late-onset wheeze was classified in both cohorts with minimal symptoms before age 3 to 3.5 years but wheezing after 6 years. These patients had a high prevalence of atopy by skin prick test $(55.7 \%)$ but did not have significantly elevated IgE levels. There was a positive association for late-onset wheezing with chronic rhinitis, maternal asthma, and male sex. Late-onset wheeze was seen in $15 \%$ of patients in the Tucson study but only $6 \%$ in the ALSPAC group and suggests a pre-asthma phenotype with symptoms stemming from asthma as opposed to viral etiology or transient small airways [17].

Persistent wheeze comprises $6.9 \%$ to $13.7 \%$ of these cohorts and is characterized by high $\operatorname{IgE}$ at 9 months with risk factors of eczema, chronic rhinitis, maternal 
asthma and smoking, Hispanic ethnicity, and male sex. This phenotype displays normal lung function early but has significantly decreased $\mathrm{V}_{\max } \mathrm{FRC}$ (maximum velocityforced residual capacity) by age 6 years. Citing the weaker association with atopy, Henderson et al. [16] suggested that this phenotype may be a mix of anatomic predilection to early wheeze combined with tendency toward asthma later in life.

\section{Inflammatory Markers}

Eosinophils traditionally have been perceived as markers of asthma. Findings that show eosinophils not present in $20 \%$ to $30 \%$ of steroid-naïve asthma patients and $50 \%$ of symptomatic, steroid-treated asthma patients, even during exacerbations, cast doubt on eosinophils' central role [19]. Further evidence against eosinophils came with the lack of clinical improvement in asthma patients on anti-IL-5 therapy despite a significant decrease in eosinophilia. There likely are at least three distinct inflammatory subsets in asthma: eosinophil predominant, neutrophil predominant, and paucigranulocytic.

Eosinophilic asthma, the predominant subtype, is the most clearly delineated and studied. Eosinophils can be detected systemically in the serum but seem more clinically relevant locally as measured in bronchoalveolar lavage (BAL), forced sputum, or by biopsy. Eosinophils can be detected in atopic and nonatopic asthma patients at a young age. Levels correlate with flares and generally seem to respond to steroids [20]. Eosinophilic asthma patients tend to respond well to steroids and bronchodilators but have a high frequency of flares $[19,21]$. This subtype also includes ASA-sensitive patients as well as some with fixed airway obstruction [21].

Eosinophilia despite appropriate corticosteroid therapy can be seen in a subset of severe, refractory asthma patients $[20,22 \bullet \bullet]$. Therapy guided by eosinophilia count has proven effective, with cutoffs of generally less than $2 \%$ of forced sputum or BAL [22••]. Selecting for the eosinophilic asthma phenotype, two recent studies showed improvement in controlling flares among patients while they were receiving anti-IL-5 therapy $[5,6]$. Thus, identifying a potentially responsive phenotype can significantly alter the measured efficacy of targeted therapy. It is important to note that some authors have questioned the validity of the eosinophilic severe asthma phenotype [23].

Neutrophilic asthma has not been as extensively studied and thus has an unknown prevalence. Neutrophils can be seen with or without eosinophils. There is some thought that neutrophilic asthma patients have been sensitized by irritants, endotoxins, or infection, thus activating innate immunity initially. These patients show an increased level of IL-8 (CXCL8), myeloperoxidase, and leukotriene $\mathrm{B}_{4}$ in $\mathrm{BAL}$ or induced sputum that is consistent with inflammation from irritants and infection or possibly from high-dose steroids [21]. Possible mechanisms for activation of innate immunity include biofilm formation, endotoxin exposure, or infection with Chlamydia or Mycoplasma spp. These patients may respond to treatment of these pathogens with macrolides but generally respond poorly to steroids [24].

The final subset has been labeled paucigranulocytic and can contain a mixed cellular presentation but with minimal infiltration. This phenotype is generally milder and may represent sampling error. However, poor sampling is less likely, as studies seem to indicate that the marker found in one area of the lung generally seems constant throughout the airways. The low level of inflammatory cells may also be a product of aggressive steroid immunosuppression [20].

\section{Infection}

Infections clearly have a significant role in asthma patients, as they commonly trigger flares. As technology improves, the detection of viral infections in patients acutely flaring continues to increase, accounting for about $50 \%$ of adult and $80 \%$ to $85 \%$ of pediatric attacks [24]. The role of early infection on the subsequent development of asthma is much less clear, with evidence indicating that viral infections can be protective against atopy or may contribute to asthma development. As we continue to observe cohorts of early wheeze, data shift from remission or resolution in adolescents to potential adult recurrence. Viral infections are the most common causes of flares and initially seemed to be the culprits in the etiology of asthma. However, data continue to build supporting atypical colonization or infection leading to proinflammatory atopic state $[25,26]$.

The most common causes of respiratory tract infection in adults include rhinovirus, coronavirus, adenovirus, influenza, respiratory syncytial virus (RSV), and human metapneumovirus, although bocavirus has been detected more commonly recently [24]. RSV seemed the most likely cause of wheezing and persistent asthma initially. A recent cohort study concluded that whereas RSV acutely increases bronchial hyperreactivity, the effect is not sustained, although asthma patients are more likely to have a severe RSV episode [27]. Focus has shifted toward rhinovirus, the most common cause of respiratory infections but a potentially greater contributor to future wheeze in patients $[28,29]$. Although the prevalence of early wheeze may be higher in RSV, rhinovirus-infected children are two to three times more likely to have wheeze at 3 years [28]. Confounding our studies of causality in viral infection may be the baseline prewheezing phenotype, with future wheeze more reliably predicted by early-transient or late-persistent wheezing phenotype. Studies continue to explore whether viral infections are drivers of asthma or simply markers of latent atopics.

One potential explanation for previous ambiguous studies on rhinovirus is the discovery of a new group of rhinovirus: human rotavirus (HRV) $\mathrm{C}$, which appears to be more strongly associated with asthma than HRV A, the more virulent of the two previous types. HRV C was 
responsible for $50 \%$ of asthma admissions associated with rhinovirus in that study [30]. Coronavirus and parainfluenza also seem implicated in a significant number of asthma patients, with lower severity than rhinovirus and RSV. Although it is confined to winter epidemics, influenza has a significant rate of fatality among asthma patients [24]. Human metapneumovirus and bocavirus have been more commonly detected and likely significantly increase in severity when contracted with another virus [24].

Although bacteria traditionally have not been thought to contribute significantly to asthma flares, more recent evidence indicates that chronic bacterial colonization may contribute to the development or unmasking of asthma as well as acute flares [31]. Atypical bacteria, including Chlamydia pneumoniae and Mycoplasma pneumoniae, are more prevalent in asthma patients, especially during acute flares, with evidence of prolonged presence (by polymerase chain reaction) up to 200 days after initial infection [24,32]. The obligate intracellular nature of Chlamydia may contribute to its chronic low-virulence nature. Multiple studies have examined treatment of asthma patients with antibiotics, with varying results on lung function and asthma symptoms [26,33]. In a study of severe, nonatopic adult asthma patients, Chlamydia was found in two thirds of patients by enzyme-linked immunosorbent assay and associated with a fourfold greater rate of decline in lung function [34]. Thus, a persistent, mild infection by atypical bacteria may cause a proinflammatory state that leads to airway remodeling and increased sensitivity to atopy. Indeed, in one murine model, mild inoculation of Chlamydia increased sensitization to allergen, whereas high doses of bacteria did not increase sensitization [35].

One final complication is the finding that colonization with bacteria such as Streptococcus pneumoniae and Haemophilus, Moraxella, and Staphylococcus spp increased the likelihood of asthma in early childhood in the Childhood Origins of Asthma Trial [25,35]. In this cohort, asymptomatic neonates observed to 5 years of age were cultured at 1 month. Those colonized with bacteria were more likely to wheeze, be hospitalized, and have a diagnosis of asthma at 5 years of age. The population-attributable risk for colonization was a significant $4.6 \%$. As our detection methods become more accurate, the detected prevalence of bacteria and viruses increases. Although to this point we have not clearly identified the population of asthma patients that benefits from antibiotics, data indicate that such a population exists.

\section{Aspirin Sensitivity}

The best defined of the triggered asthma phenotypes may be Samter's triad, which is characterized by nasal polyposis, ASA sensitivity, and asthma. Although it has been described in the literature for decades, the current prevalence of $14 \%$ in a recent study is likely confounded by underdiagnosis [36]. This phenotype is more common in severe asthma patients, especially females, and is likely caused by eosinophilic-activated remodeling of the airways with chronic nonatopic eosinophilic sinusitis [37]. It is often seen in adults at presentation, as refractory chronic perennial rhinitis precedes ASA/NSAID sensitivity, polyposis, and worsening asthma [37]. Individuals examined from the Epidemiology and Natural History of Asthma: Outcomes and Treatment Regimens (TENOR) study population of severe asthma patients showed ASAexacerbated respiratory disease patients have lower $\mathrm{FEV}_{1}$, higher asthma severity classification, greater incidence of intubation, greater recent steroid burst, and higher use of high-dose inhaled corticosteroids than non-ASA-sensitive asthma patients [36]. Acutely, patients present with flushing, urticaria, edema, rhinitis, and often asthma flares, frequently up to 3 hours after exposure [37].

ASA-induced symptoms are thought to be non- $\operatorname{IgE}$ mediated. Inhibition of cyclooxygenase- 1 leads to lower levels of prostaglandin $\mathrm{E}_{2}$, a noted anti-inflammatory mediator that can block cysteinyl leukotrienes. Studies have confirmed a higher level of cysteinyl leukotrienes in ASA-sensitive patients, specifically leukotriene $\mathrm{C}_{4} \mathrm{~S}$ with promoter $-444 \mathrm{C}$, the level of which is higher in Japanese populations $[38,39]$. The level of urinary leukotriene $\mathrm{E}_{4}$ generally is significantly higher than it is in non-ASA-sensitive patients [37]. These patients have shown significant improvement on leukotriene modifiers and with ASA desensitization, nasal polypectomy, and topical steroids. The inflammatory progression and possible airway remodeling continue regardless of exposure history to ASA. A recent study suggested that ASA desensitization for severe refractory asthma is also cost-effective [40].

The ASA-exacerbated respiratory disease phenotype falls into that of eosinophilic and severe asthma. It offers a unique classification in that several interventions have proven effective in lowering asthma morbidity. Depending on the level of polyposis, surgery initially may be indicated, followed by desensitization and maintenance doses, as well as leukotriene modifiers (eg, a lipoxygenase inhibitor such as zileuton). Desensitization appears to lower morbidity regardless of prior exposure [37].

\section{Exercise}

The phenotype of exercise-induced asthma (EIA) is also likely an umbrella term, with significant differential diagnoses that in many studies have exceeded asthma in incidence upon testing. EIA was defined by the American Academy of Allergy, Asthma, and Immunology working group as "the condition in which exercise induces symptoms of asthma in patients who have asthma," whereas exercise-induced bronchospasm does not require a diagnosis of asthma [41•]. Various mechanisms have been postulated for EIA, including hyperventilation leading to increased irritant cold dry air exposure and subsequent desiccation of the airways $[41 \bullet, 42]$. Further inflammation can lead to activation of neutrophils, mast cells, and 
proinflammatory cytokines, causing significant inflammation. The prevalence of EIA is about $5 \%$ to $20 \%$.

Symptoms of EIA include dyspnea, chest pain or tightness, wheeze, and cough. These symptoms should be accompanied by a reversible drop in $\mathrm{FEV}_{1}$ of $10 \%$ to $20 \%$. In our center, we have found exercise challenge useful in this diagnosis, with several children testing positive for dehydration, vocal cord dysfunction, and cardiac arrhythmias such as prolonged QT. Other common causes of dyspnea with exercise include hyperventilation, deconditioning, gastroesophageal reflux, laryngomalacia, exercise-induced anaphylaxis, and restrictive disease from body habitus. There seems to be a predominance of elite athletes with symptoms of EIA, although some of these cases may well be misdiagnosed vocal cord dysfunction presenting soon after exercise and feeling of obstruction in the throat as well as inspiratory stridor. There appears to be a protective response of exercise that delays symptoms for 2 to 3 hours. Prophylactic $\beta$-agonist inhaler or cromolyn administered 10 to 15 minutes before exercise can also decrease symptoms [41•], although some controversy exists as to whether regular use can worsen EIA.

\section{Severe Asthma}

The phenotype of severe asthma makes up only $5 \%$ to $10 \%$ of asthma patients but is responsible for $30 \%$ to $40 \%$ of asthma's economic burden [22••]. Patients in this phenotype may be steroid dependent or resistant, which is theorized to correlate with responsive neutrophilic or nonresponsive persistent-eosinophilic inflammation. Other subtypes have been inconsistently associated, such as ASA sensitivity; older, obese, menstruating female patients; and smokers. Multiple cohorts are being observed for further elucidation of the severe asthma phenotype, including the Severe Asthma Research Program (SARP), the TENOR group, and the European Network for Understanding Mechanisms of Severe Asthma study. The SARP and European Network for Understanding Mechanisms of Severe Asthma groups specifically incorporate medicine requirements in their diagnosis of severe asthma. Through these cohorts, constant variables have emerged, as has the heterogeneity of this phenotype.

The SARP, initiated by the National Heart, Lung, and Blood Institute, observed severe asthma patients as defined by the American Thoracic Society consensus, which required one of two major criteria (majority of year with oral corticosteroids and high-dose inhaled corticosteroids) as well as two of seven minor criteria (additional controller, near-daily short-acting $\beta$-agonist use, $\mathrm{FEV}_{1}<$ $80 \%$, one or more urgent care visits, three or more oral steroid bursts, rapid deterioration with $<25 \%$ decrease in steroid dose, and intubation). General characteristics found among severe asthma patients were older age and longer duration of disease, more daily symptoms of dyspnea and cough, higher utilization rates, lower lung function (that was reversible), less atopy, and history of pneumonia [22••]. Within SARP, there was no significance of sex or body mass index, although other studies reported female sex and obesity as a distinct cluster in this phenotype [7,43]. Not surprisingly, severe asthma was most consistently distinguished by three oral steroid bursts and intubation. Also critical was the finding that these patients were predominantly reversible and had significant epithelial and lamina reticularis thickening that was potentially triggered by chronic infection $[22 \bullet \bullet]$.

Severe asthma has been defined by early and late onset that is generally delineated by onset before or after 12 years of age. Early onset generally is more atopic with longer duration of disease and, in one study, lower urinary leukotriene $\mathrm{E}_{4}$ and less eosinophilia [4]. Late onset is less common, with higher eosinophilia and lower lung $\mathrm{FEV}_{1}$ despite shorter duration. Some have theorized that the persistent eosinophilia despite chronic steroid use indicated nonresponsiveness [20]. In a study of severe asthma patients, however, ten Brinke et al. [23] were able to lower eosinophils to undetectable levels with 2 weeks of intramuscular triamcinolone and to improve $\mathrm{FEV}_{1}$ while decreasing rescue therapy. Thus, the classification of eosinophilic severe asthma is still controversial and may identify a population that can be controlled with appropriate aggressive treatment. Other common characteristics found in severe asthma patient cohorts include ASA sensitivity, gastroesophageal reflux disease, psychological dysfunctioning, and sinopulmonary disease $[22 \bullet, 44]$. Treatment possibilities include multiple immunomodulators, with some success seen with anti-IgE and anti-IL-5 recently, although a trial of anti-tumor necrosis factor- $\alpha$ did not show clinical improvement $[5,6]$.

\section{Flare Prone}

The greatest cost of asthma to the patient's future lung function and to the health care system stems from severe exacerbations that often require emergency department visits and hospitalizations. Although severe asthma patients with difficult-to-control disease account for many of these visits, it is clear that asthma patients of all baseline severity can have flares that cause significant and persistent damage [45]. Indeed, in the most recent National Heart, Lung, and Blood Institute guidelines, the concept of risk is added to the traditional daily impairment classification of asthma. Although a patient may have complete control between flares, repeated emergency department visits or hospitalizations indicate inadequate control and therefore raise the patient's severity classification [1]. The importance of preventing flares was emphasized by a recent study that estimated a $30.2-\mathrm{mL}$ greater annual decline in $\mathrm{FEV}_{1}$ [46]. Because asthma patients of all severity levels can have frequent flares, attempts have been made to describe this population of "flare-prone" patients. Whereas multiple studies have examined risk factors for exacerbations, only a few have attempted to characterize the population of frequent-flaring asthma patients. Risk 
factors include female sex, previous flare, recurrent infections, and allergen exposure.

Multiple risk factors have been found in various studies, although somewhat inconsistently. One constant risk factor for frequent flares is previous exacerbation, which can have an OR as high as $6.33[47,48]$. Other risk factors for flares include lower baseline $\mathrm{FEV}_{1}$, severe nasal sinus disease, and psychological dysfunction [46,47,49]. Most flares are caused by infections, with a pathogen found in as many as $80 \%$ of acutely flaring asthma patients [50]. Rhinovirus has been found in about two thirds of infection-triggered patients, and infection severity seems to correlate with flare severity [50]. Given that asthma patients seem to have a higher susceptibility to infection, possibly caused by defects in innate immunity and interferon- $\gamma$ cytokine production, flare-prone asthma patients may have a more significant defect that leads to frequent infections and thus exacerbations [50]. Finally, one recent study estimated that exposure of an asthma patient to an infection and an allergen trigger can increase the OR of flare to 19.4 (95\% CI, 3.1-101.5) [51]. Further elucidating the phenotype that is more likely to have severe flares regardless of baseline symptoms may significantly increase future $\mathrm{FEV}_{1}$ and the burden of asthma [16].

\section{Conclusions}

Although this review is not exhaustive of all asthma syndromes, it includes most of those with chronic, variably reversible obstruction and hyperreactivity. More precise phenotypic classification is essential for directing aggressive intervention for selected asthmatic populations and effective treatment for responders. The process would be aided by advances in molecular pathogenesis and diagnosis.

\section{Disclosure}

No potential conflicts of interest relevant to this article were reported.

\section{References and Recommended Reading}

Papers of particular interest, published recently, have been highlighted as:

- Of importance

• Of major importance

1. National Institutes of Health, National Heart, Lung, and Blood Institute. National Asthma Education and Prevention Program. Expert Panel Report 3: Guidelines for the Diagnosis and Management of Asthma. Bethesda, MD: National Heart, Lung, and Blood Institute; 2007.

2. Bouzigon E, Corda E, Aschard H, et al.: Effect of 17q21 variants and smoking exposure in early-onset asthma. $N$ Engl J Med 2008, 359:1985-1994.

3. Leckie MJ, ten Brinke A, Khan J, et al.: Effects of an interleukin-5 blocking monoclonal antibody on eosinophils, airway hyper-responsiveness, and the late asthmatic response. Lancet 2000, 356:2144-2148.
4. Miranda C, Busacker A, Balzar S, et al.: Distinguishing severe asthma phenotypes: role of age at onset and eosinophilic inflammation. I Allergy Clin Immunol 2004, 113:101-108.

5. Haldar P, Brightling CE, Hargadon B, et al.: Mepolizumab and exacerbations of refractory eosinophilic asthma. $N$ Engl J Med 2009, 360:973-984.

6. Nair P, Pizzichini MM, Kjarsgaard M, et al.: Mepolizumab for prednisone-dependent asthma with sputum eosinophilia. N Engl J Med 2009, 360:985-993.

7. Haldar P, Pavord ID, Shaw DE, et al.: Cluster analysis and clinical asthma phenotypes. Am J Respir Crit Care Med 2008, 178:218-224.

8. Arbes SJ Jr, Gergen PJ, Vaughn B, Zeldin DC: Asthma cases attributable to atopy: results from the Third National Health and Nutrition Examination Survey. J Allergy Clin Immunol 2007, 120:1139-1145.

9. Ahern D, Lloyd CM, Robinson DS: Chemokine responsiveness of CD4+ CD25+ regulatory and CD4+ CD25-T cells from atopic and nonatopic donors. Allergy 2009, 64:1121-1129.

10. Drews AC, Pizzichini MM, Pizzichini E, et al.: Neutrophilic airway inflammation is a main feature of induced sputum in nonatopic asthmatic children. Allergy 2009 Apr 14 (Epub ahead of print).

11. Romanet-Manent S, Charpin D, Magnan A, et al.: Allergic vs nonallergic asthma: what makes the difference? Allergy 2002, 57:607-613.

12. Novak N, Bieber T: Allergic and nonallergic forms of atopic diseases. J Allergy Clin Immunol 2003, 112:252-262.

13. Turato G, Barbato A, Baraldo S, et al.: Nonatopic children with multitrigger wheezing have airway pathology comparable to atopic asthma. Am J Respir Crit Care Med 2008, 178:476-482.

14. Ownby DR, Joseph CL: Should nonatopic asthma get equal attention? J Allergy Clin Immunol 2007, 120:1018-1020.

15. Sears MR, Greene JM, Willan AR, et al.: A longitudinal, population-based, cohort study of childhood asthma followed to adulthood. N Engl J Med 2003, 349:1414-1422.

16. Henderson J, Granell R, Heron J, et al.: Associations of wheezing phenotypes in the first 6 years of life with atopy, lung function and airway responsiveness in mid-childhood. Thorax 2008, 63:974-980.

17. Martinez FD, Wright AL, Taussig LM, et al.: Asthma and wheezing in the first six years of life. The Group Health Medical Associates. N Engl J Med 1995, 332:133-138.

18. Strachan DP, Butland BK, Anderson HR: Incidence and prognosis of asthma and wheezing illness from early childhood to age 33 in a national British cohort. BMJ 1996, 312:1195-1199.

19. Haldar P, Pavord ID: Noneosinophilic asthma: a distinct clinical and pathologic phenotype. I Allergy Clin Immunol 2007, 119:1043-1052; quiz 1053-1054.

20. Wenzel SE: Asthma: defining of the persistent adult phenotypes. Lancet 2006, 368:804-813.

21. Borish L, Culp JA: Asthma: a syndrome composed of heterogeneous diseases. Ann Allergy Asthma Immunol 2008, 101:1-8; quiz 8-11, 50.

22.• Moore WC, Bleecker ER, Curran-Everett D, et al.: Characterization of the severe asthma phenotype by the National Heart, Lung, and Blood Institute's Severe Asthma Research Program. J Allergy Clin Immunol 2007, 119:405-413.

This paper is an important review of findings from the SARP severe asthma cohort formed by the National Heart, Lung, and Blood Institute.

23. ten Brinke A, Zwinderman AH, Sterk PJ, et al.: "Refractory" eosinophilic airway inflammation in severe asthma: effect of parenteral corticosteroids. Am J Respir Crit Care Med 2004, 170:601-605.

24. MacDowell AL, Bacharier LB: Infectious triggers of asthma. Immunol Allergy Clin North Am 2005, 25:45-66.

25. Bisgaard H, Hermansen MN, Buchvald F, et al.: Childhood asthma after bacterial colonization of the airway in neonates. N Engl J Med 2007, 357:1487-1495. 
26. Kraft M, Cassell GH, Pak J, Martin RJ: Mycoplasma pneumoniae and Chlamydia pneumoniae in asthma: effect of clarithromycin. Chest 2002, 121:1782-1788.

27. Stensballe LG, Simonsen JB, Thomsen SF, et al.: The causal direction in the association between respiratory syncytial virus hospitalization and asthma. J Allergy Clin Immunol 2009, 123:131.e1-137.e1.

28. Lemanske RF Jr, Jackson DJ, Gangnon RE, et al.: Rhinovirus illnesses during infancy predict subsequent childhood wheezing. J Allergy Clin Immunol 2005, 116:571-577.

29. Kusel MM, de Klerk NH, Kebadze T, et al.: Early-life respiratory viral infections, atopic sensitization, and risk of subsequent development of persistent asthma. J Allergy Clin Immunol 2007, 119:1105-1110.

30. Miller EK, Edwards KM, Weinberg GA, et al.: A novel group of rhinoviruses is associated with asthma hospitalizations. J Allergy Clin Immunol 2009, 123:98.e1-104.e1.

31. Sutherland ER, Martin RJ: Asthma and atypical bacterial infection. Chest 2007, 132:1962-1966.

32. Lieberman D, Printz S, Ben-Yaakov M, et al.: Atypical pathogen infection in adults with acute exacerbation of bronchial asthma. Am J Respir Crit Care Med 2003, 167:406-410.

33. Johnston SL, Blasi F, Black PN, et al.: The effect of telithromycin in acute exacerbations of asthma. N Engl J Med 2006, 354:1589-1600.

34. ten Brinke A, van Dissel JT, Sterk PJ, et al.: Persistent airflow limitation in adult-onset nonatopic asthma is associated with serologic evidence of Chlamydia pneumoniae infection. J Allergy Clin Immunol 2001, 107:449-454.

35. Schroder NW, Crother TR, Naiki Y, et al.: Innate immune responses during respiratory tract infection with a bacterial pathogen induce allergic airway sensitization. J Allergy Clin Immunol 2008, 122:595.e5-602.e5.

36. Mascia K, Haselkorn T, Deniz YM, et al.: Aspirin sensitivity and severity of asthma: evidence for irreversible airway obstruction in patients with severe or difficult-to-treat asthma. J Allergy Clin Immunol 2005, 116:970-975.

37. Szczeklik A, Stevenson DD: Aspirin-induced asthma: advances in pathogenesis, diagnosis, and management. J Allergy Clin Immunol 2003, 111:913-921; quiz 922.

38. Kawagishi Y, Mita H, Taniguchi M, et al.: Leukotriene C4 synthase promoter polymorphism in Japanese patients with aspirin-induced asthma. J Allergy Clin Immunol 2002, 109:936-942.
39. Sousa AR, Parikh A, Scadding G, et al.: Leukotrienereceptor expression on nasal mucosal inflammatory cells in aspirin-sensitive rhinosinusitis. N Engl J Med 2002, 347:1493-1499.

40. Shaker M, Lobb A, Jenkins P, et al.: An economic analysis of aspirin desensitization in aspirin-exacerbated respiratory disease. J Allergy Clin Immunol 2008, 121:81-87.

41. Weiler JM, Bonini S, Coifman R, et al.: American Academy of Allergy, Asthma \& Immunology Work Group report: exercise-induced asthma. J Allergy Clin Immunol 2007, 119:1349-1358.

This is a full review of exercise-induced bronchospasm.

42. Fitch KD, Sue-Chu M, Anderson SD, et al.: Asthma and the elite athlete: summary of the International Olympic Committee's consensus conference, Lausanne, Switzerland, January 22-24, 2008. J Allergy Clin Immunol 2008, 122:254-260, 260.e1-260.e7.

43. Lessard A, Turcotte H, Cormier Y, Boulet LP: Obesity and asthma: a specific phenotype? Chest 2008, 134:317-323.

44. Brinke T: Risk factors of frequent exacerbations in difficultto-treat asthma. Eur Respir J 2005, 26:812-818.

45. Carroll CL, Schramm CM, Zucker AR: Severe exacerbations in children with mild asthma: characterizing a pediatric phenotype. J Asthma 2008, 45:513-517.

46. Bai TR, Vonk JM, Postma DS, Boezen HM: Severe exacerbations predict excess lung function decline in asthma. Eur Respir J 2007, 30:452-456.

47. ten Brinke A, Sterk PJ, Masclee AA, et al.: Risk factors of frequent exacerbations in difficult-to-treat asthma. Eur Respir J 2005, 26:812-818.

48. Miller MK, Lee JH, Miller DP, Wenzel SE: Recent asthma exacerbations: a key predictor of future exacerbations. Respir Med 2007, 101:481-489.

49. Tattersfield AE, Postma DS, Barnes PJ, et al.: Exacerbations of asthma: a descriptive study of $\mathbf{4 2 5}$ severe exacerbations. The FACET International Study Group. Am J Respir Crit Care Med 1999, 160:594-599.

50. Sykes A, Johnston SL: Etiology of asthma exacerbations. Allergy Clin Immunol 2008, 122:685-688.

51. Murray CS, Poletti G, Kebadze T, et al.: Study of modifiable risk factors for asthma exacerbations: virus infection and allergen exposure increase the risk of asthma hospital admissions in children. Thorax 2006, 61:376-382. 